\title{
General Correlation of Pressure Distribution for Swirl Flow in Pipes
}

\author{
M. H. Rmbaby \\ Mech, Power Eng. Dept. Faculty of Eng., \\ Menoufia Univ., Egypt.
}

\begin{abstract}
Early correlations of wall static pressure distribution along pipes, through which a Newtonian fluid flows with-unguided vane generated-swirl component, have been generalized. Generalization includes another - tangential tap generated swirl type in addition to fluids other than Newtonian. These correlations are of great benefit for many applications provided knowledge of the fluid rheological properties as well as the conventional friction factor correlations. An expression for swirl number evaluation has been introduced in order to make use of the available correlations. Application on non-Newtomian power law fluids has been carried out and the correlation represented well the available experimental data for both laminar and turbulent flow conditions. Application on drag reduction flow, under conditions of maximum drag reduction, has shown that the percentage drag reduction increases as the pipe length increases, i.e., as the swirl intensity decreases.
\end{abstract}

Manuscript Received From Dr. M.H.Embaby on: 22/7/1998.

Accepted on: 15/9/1998.

Engineering Research Bulletin, Vol. 22, No.1, 1999.

Minufiya University, Faculty of Engineering, Shebin El-Kom, EGYPT, iss. 1110-1180. 


\section{INTRODUCTION}

Problems related to swirl flow systems have been found in several applications. Among these applications, there are heat transfer enhancement in heat exchangers, fuel burners, raw material purification processes and many fluidic devices. Swirl may be generated by many techniques such as rotating a section of test pipe, twisted taps, coiled wires and entry vanes. Swirl flow may be guided, as in the case of coiled pipes, or unguided one. The later flow, though more complicate to analyse, is widely met in practice. The present work deals with problems of unguided swirl flow of some practically important fluids such as non-Newtonian power law fluids and drag reducing ones.

Review of previous work has shown that the Newtonian unguided swirl flow in pipes has been studied by many authors [1-8]. These studies have shown that the swirl decays exponentially and that each of the pressure distribution, velocity profiels and turbulence characteristics are affected by swirl. Experimental investigations of turbulent swirl flow through pipes are numerous [1-8]. These investigations were mainly concerned with axial velocity profiles, tangential velocity profiles, and decay of swirl. However, Hui Li et al [7] studied experimentally swirl decay, the average dynamic, static and total pressure distributions along a pipe. Flow Reynolds numbers were 60,00 and 125,000. Based on their-and earlier-results, they established empirical correlations for the above flow characteristics.

Although turbulent swirl flow through a pipe is a highly complex one, some analytical and theoretical approaches have appeared. For example, Krieth et. al. [1] simplified the governing equations using an order of magnitude analysis and assumed a constant eddy viscosity. Swirl decay results agreed well with the experimental ones. On the other hand, a quilititative agreement was obtained for the velocity distributions. El Shorbagy [2] adapted a different integral technique using suitable simplifying assumptions. Friction factor distributions along a pipe were predicted and confirmed by comparison with experimental results. Recently , Parchen et al. [9] gave numerical predictions which were based on a standard $\mathrm{k}-\varepsilon$ model and models based on an algebraic transport model for the turbulent stresses. Predictions included velocity profiles and swirl decay while pressure distributions were not given.

Some studies have concerned with the phenomenon of vortex break down [e.g 9], while others [e.g. 10] have concerned with turbulence characteristics for turbulent swirl flows.

Studies on non-Newtonian power law fluid swirl flow in pipes seem to be little. For example, Dostal et.al. [11] presented numerical solution for the helical flow of a power law fluid between two cylinders with rotating the inner one. It is noticed that this study was restricted on laminar flow and the results cann't be generalized to other unguided swirl flows. 
Salem [12] studied experimentally the laminar and turbulent non-Newtonian tangential tap generated swirl flow in a pipe. Also, he extended an earlier theoretical integral technique-adapted with Newtonian fluids [2] - to nonNewtonian power law fluids under turbulent swirl flow conditions. Comparison between theory and experimental showed a qualitative agreement only.

The above review shows that there is a need for simple correlations, like those recently available for Newtonian fluids [7] to be adapted with other fluids. This has been the objective of the present work. Thus, similar correlations have been derived and a discussion for applications on some known fluids such as power law and drag reducing fluids has been presented.

\section{ANALYSIS}

Hui $\mathrm{Li}$ et.al. [7] had correlated the pressure distribution data for a guide vane generated unguided swirl turbulent flow of a Newtonian fluid through a pipe. The wall static pressure distribution correlation was given as :

$$
\begin{aligned}
& \Delta \mathrm{P}_{\mathrm{x}}=\Delta \mathrm{P}_{\mathrm{s}}+\Delta \mathrm{P}_{\mathrm{a}} \\
& \Delta \mathrm{P}_{\mathrm{a}}=\lambda(\mathrm{L}-\mathrm{x}) \\
& \Delta \mathrm{P}_{\mathrm{s}}=0.745 \mathrm{~S}+4.16 \mathrm{~S}^{2}
\end{aligned}
$$

where $\Delta \mathrm{P}_{\mathrm{x}}$ is the dimensionless wall static pressure drop, $\Delta \mathrm{P}_{\mathrm{s}}$ is the dimensionless pressure drop due to swirl, $\Delta \mathrm{P}_{\mathrm{a}}$ is the dimensionless pressure drop due to axial flow ; $\lambda$ is the conventional axial flow friction coefficient defined as:

$$
\lambda=8 \tau_{\mathrm{w}} / \rho u_{\mathrm{nn}}{ }^{2}
$$

and $\mathrm{S}$ is a swirl number defined as :

$$
S=\frac{\int_{0}^{1} u w r^{2} d r}{\int_{0}^{1} u^{2} r d r}
$$

which is a measure of swirl intensity at a section of distance $x$ from pipe inlet.$L$ is the dimensionless pipe length.

The parameter $\mathrm{S}$ above, was determined by measuring both the axial velocity component " $u$ " and the tangential one " $w$ " at different pipe sections. The results were correlated and the swirl decay was given in [7] by :

$$
\log \left(S / S_{0}\right)=-0.01605 x^{0.8}
$$

where $S_{0}$ is the swirl parameter at inlet $\mathrm{x}=0$

Inspection of Eqs. (1-3) shows that $\Delta P_{\mathrm{a}}$ depends on the conventional friction factor correlations of the fully developed flow. On the other hand; the contribution due to swirl flow, namely $\Delta P_{\mathrm{s}}$ depends on the swirl intensity $\mathrm{S}$ in addition to other parameters of the fluid - pipe system. The following section has 
two objectives. The first is to obtain an expression for the swirl intensity $\mathrm{S}$ for the present tap injection swirl generated system. The second is to generalize the correlations ( 3 ) and (6) by application on both non-Newtonian power law fluids and drag reducing ones.

\subsection{Evaluation of the swirl intensity(S)}

Equation (5), shows that determination of the parameter $\mathrm{S}$ requires evaluation of both axial and tangential velocity profiles.

\subsubsection{Axial Velocity Profiles}

It is assumed that the jet attaches to the pipe wall at a neglegible distance downstream of the inlet section [13]. In addition, earlier studies $[1,3,4]$ had shown that the wall law was obeyed except at relatively high swirl intensities ( $S$ $>1.0$ ). This means that the assumption of wall law profile for the axial component of the flow velocity should be anomalous near pipe inlet. On the other hand, the deviation between the assumed and real profiles decreases in the downstream direction where swirl intensity continuously decreases. Thus, the following profiles for the axial component are adapted.

\section{(A) - Non-Newronian Power Law Fluids:}

For a power law fluid flow through pipes the axial velocity profiles are given by [14]:

$$
\begin{aligned}
& \text { laminar flow : } u=\alpha\left(1-r^{\beta}\right) \\
& \text { where } \quad \alpha=\frac{3 n+1}{n+1} ; \quad \beta=\frac{n+1}{n} \\
& \text { turbulent flow: } \quad u^{+}=\left(y^{+}\right)^{1 / n} \quad ; \quad y^{+} \leq 5^{n} \\
& \mathrm{u}^{+}=\mathrm{A} \ln \mathrm{y}^{+}+\mathrm{B} ; \quad \mathrm{Y}^{+}>5^{\prime \prime} \\
& \text { where : } u^{+}=u^{\prime} / u^{*}, y^{+}=\rho y^{n} u^{*}(2-n) / \eta_{0} \text {, } \\
& u^{*}=\sqrt{\tau_{w} / \rho} \\
& A=2.46 / \mathrm{n}^{0.75} \\
& B=3.75\left(1.96+0.8 \ln -0.7 \ln (3+1 / \mathrm{n}) / \mathrm{n}^{0.75}-0.566 / \mathrm{n}^{1.2}\right.
\end{aligned}
$$




\section{B- Drag Reduction Flow:}

Drag reduction may be caused due to polymer additives, soap solutions, fiber suspension etc. [15] under turbulent flow conditions. It had been indicated that these flows had a maximum drag reduction (mdr) asymptote. This asymptotic condition will be considered for aim of comparison with the Newtonian flow case. Therefore, under (mdr) conditions the velocity profile is given by [15]:

$$
\begin{array}{lll}
\mathrm{u}^{+}=\mathrm{y}^{+} & ; & \mathrm{y}^{+} \leq 11.7 \\
\mathrm{u}^{+}=11.7 \ln \mathrm{y}^{+}-17 & ; & \mathrm{y}^{+}>11.7
\end{array}
$$

\subsubsection{Tangential Velocity Profiles}

Earlier studies on unguided swirl flow $[1,5]$ have shown that the tangential velocity profiles are characterised by three zones. These are (i) a wall layer with a steep gradient, (ii) an annular free vortex type zone and (iii) a forced vortex core. The size of each zone depends on the swirl intensity with the maximum value being continuously decaying in the down stream direction. The later study [5] showed that there had been some deviations between the prementioned profiles and the measured ones, especially in the core zone. In this zone, a flat portion around the pipe center line prevails to a certain extent. However, to facilitate analysis, the following assumptions [12] are adapted. Figure (1) shows a sketch for the swirl generating system considered. A jet of cross section " $\mathrm{a}_{5}$ " flows into the entrance of the main pipe, of cross section area "a", with an inclination " $\theta$ " to the axial direction. The mean ejection velocity is $V^{\prime} J$ while the axial mean velocity in the pipe is $u_{m}$. By continuity, thus:

$$
\mathrm{V}_{\mathrm{J}}=\frac{\mathrm{a}}{\mathrm{a}_{\mathrm{J}}}
$$

The tangential velocity component " $W_{0}$ " is then given by:

$$
w_{0}=v_{J} \sin \theta=\frac{a}{a_{J}} \sin \theta ; \quad \theta<90^{\circ}
$$

It is worthy to note that (i) this expression for $W_{0}$ satisfies the continuity requirements and (ii) it is still valid for all values of $\theta$. These conditions; (i) and (ii), were not satisfied when the assumption that $W_{0}=V_{J} \tan \theta$ was adapted in earlier studies [12]. It is then assumed that when the jet attaches to the pipe wall, the tangential velocity profile consists of a negligible wall layer, a free vortex and a forced vortex zones [see Fig. (1)]. In addition, it is assumed that these zones have the following characteristics:

(i) the free vortex profile extends from the pipe wall to the center line of the ejector i.e to a distance $\delta{ }^{\prime}$ from pipe wall where the velocity is a maximum "W".

(ii) the core, forced vortex zone, extends from the ejector center line to the pipe center line. 
Accordingly, the tangential velocity profile is assumed to be given by:

forced vortex core $: \mathrm{w}=\mathrm{Wr} \quad ; \quad 0 \leq \mathrm{r} \leq 1-\delta_{\mathrm{J}}$

free vortex zone $\quad: w=w\left(\frac{1-r}{\delta_{\mathrm{J}}}\right) ; \quad 1-\delta_{\mathrm{J}} \leq r \leq 1$

The above profile is assumed for both power law and drag reducing fluids.

\subsubsection{Axial Flow Friction Factor}

For power law fluids, the friction factor " $\mathrm{f}$ " $-\mathrm{f}=\lambda / 4$ - is correlated using a generalized Reynolds number given by [14]:

$$
R e=\frac{\rho D^{n} u_{m}{ }^{2-n}}{\eta_{0} 8^{(n-1)}}
$$

For laminar flow, the friction factor "p" is given by :

$$
f=16 / \operatorname{Re}
$$

For turbulent flows, the $\mathrm{f}-\mathrm{Re}$ relations are obtained by integrating the velocity profiles, Eqs. $(9,10)$ for power law fluids and Eq. (14) for (mdr), across the pipe cross section. This results in equations in the form :

$$
1 / \sqrt{\mathrm{f}}=A_{0} \ln \left(\mathrm{R}_{e}{ }^{G_{1}} \mathrm{f}^{G_{2}}\right)+B_{0}
$$

The parameters $A_{0}, B_{0}, G_{1}$ and $G_{2}$ are given by :

(i) power law fluids:

$$
\begin{aligned}
& A_{0}=n A / \sqrt{2} \\
& B_{0}=\left[(n-1) A \ln 8+\frac{(n-2)}{2} A \ln 2-2.19 n A+B\right] / \sqrt{2} \\
& G 1=1 / n ; \quad G_{2}=(2-n) / 2 n
\end{aligned}
$$

(ii) maximum drag reduction (mdr):

$$
A_{0}=8.27: B_{0}=-33 ; \quad G_{1}=1 ; \quad G_{2}=0.5
$$

\subsubsection{Evaluation of Inlet Swirl Intensity "\$ $\mathrm{S}_{0}$ "}

As the axial and tangential velocity profiles have been obtained, Eq. (5) can be used for obtaining the swirl intensity at inlet $S_{0}$ as follows.

(i) Laminar power law fluid flow:

Substituting from Eqs. (7) - for the axial component and Eqs. (17-18) for the tangential one into eq. (5) gives:

$$
\begin{aligned}
S_{0}=W_{0} A_{1} / B_{1} & \\
A_{1}= & \frac{\varphi^{4}}{4}-\frac{\varphi^{\beta+4}}{\beta+4}=\left[\frac{1-\varphi^{3}}{3}-\frac{\left(1-\varphi^{4}\right)}{4}-\frac{\left(1-\varphi^{\beta+3}\right)}{\beta+3}+\frac{\left(1-\varphi^{\beta+4}\right)}{\beta+4}\right] \frac{1}{\delta_{J}}
\end{aligned}
$$




$$
B_{1}=\alpha\left[0.5-\frac{2}{\beta+2}+\frac{1}{2 \beta+2}\right]
$$

where $\varphi=1-\delta$, while $\alpha$ and $\beta$ are given in Eq. (8).

(ii) Turbulent power law - or drag reducing fluid flow :

Eqs. (10) and (14) can be written in the form :

$$
\mathrm{u}=\mathrm{Z}_{1} \ln \mathrm{y}+\mathrm{Z}_{2}
$$

where $y=y^{2} / R$

for power law fluids : $z_{1}=n A \sqrt{f / 2}$

$$
z_{2}=A \sqrt{\frac{f}{2}} \ln \left\{2^{2 n-3} \operatorname{Re}\left(\frac{f}{2}\right)^{\frac{2-n}{2}}\right\}+B
$$

for (mdr) flow: $\quad Z_{1}=11.7 \sqrt{\mathrm{f} / 2}$

$$
z_{2}=11.7 \sqrt{f / 2} \ln \left\{\frac{1}{2} \operatorname{Re} \sqrt{\frac{f}{2}}\right\}-17.0
$$

substituting from (26) and (17-18) into (5) gives :

where

$$
\mathrm{S}_{\mathrm{o}}=\mathrm{W}_{\mathrm{o}} \mathrm{A}_{2} / \mathrm{B}_{2}
$$

$$
\begin{gathered}
A_{2}=u_{J} \delta_{J}\left(-0.5+0.83 \delta_{J}-0.75 \delta_{J}^{2}+0.25 \delta_{J}^{3}\right)+ \\
Z_{1} \delta_{J}\left(0.75-0.528 \delta_{J}+0.166 \delta^{2}-0.166 \delta_{\mathrm{J}}^{3}\right) \\
\quad-0.521 Z_{1}+0.25 Z_{2} \\
B_{2}=1.75 Z_{1}^{2}-1.5 Z_{1} Z_{2}+0.5 Z_{2}^{2} \\
u_{j}=Z_{1} \ln \delta_{J}+Z_{2}
\end{gathered}
$$

\subsubsection{Generalized Correlations}

Earlier studies [12] showed that the pressure drop due to swirl $\Delta P_{\mathrm{s}} ; \mathrm{Eq}$. (3), is mainly attributed to tangential flow. This is given by :

$$
\Delta \mathrm{P}_{s}=\frac{\rho g}{\mathrm{G}} \int \tau_{\mathrm{tw}} \mathrm{w}^{{ }^{\prime}}{ }_{\mathrm{w}} \mathrm{dA} \mathrm{A}_{\mathrm{w}}
$$

where $G$ is the fluid weight flow rate $\left(G=\rho g \pi R^{2} u_{n 1}\right), w^{*}{ }_{n}$ tangential velocity at pipe wall, $\tau_{\mathrm{tw}}$ tangential component of shear stress at the wall and $d A$, is the incremental wall area $\left(\mathrm{dA}_{\mathrm{w}}=\pi \mathrm{D} d \mathrm{x}^{\prime}\right)$.

In cylindrical coordinates $\left(x, r^{\prime}, \theta\right), \tau_{t w}=\tau_{\theta r}$ which is given, in terms of the deformation tensor component $\Delta_{\mathrm{or}}$, as [15]:

$$
\tau_{\theta r}=\eta . \Delta \Delta_{\text {er }}
$$

$\eta$ is the equivalent viscosity which reduces to the Newtonian viscosity $\mu$ for a Newtonian fluid. For a power law fluid, it is given by :

$$
\eta=\eta_{0}\left[\frac{\Delta: \Delta}{2}\right]^{\frac{n-1}{2}}
$$


$\Delta$ is the deformation tensor, and the function $\left(\frac{\Delta: \Delta}{2}\right),[16]$, can be evaluated at the wall using the boundary conditions : $u=v=\frac{\partial}{\partial x}=\frac{\partial}{\partial \theta}=0$. Thus:

$$
\left.\frac{\Delta: \Delta}{2}\right|_{w} \cong\left[\left.\frac{\partial w}{\partial r}\right|_{w}\right]^{2}
$$

and

$$
\left.\Delta_{\theta r}\right|_{w} \cong \frac{\partial w}{\partial r} I_{w}
$$

According to the assumed tangential velocity profile shown in Fig. (1), thus,

$$
\left.\frac{\partial \mathrm{w}}{\partial \mathrm{r}}\right|_{\mathrm{w}} \cong \frac{\mathrm{w}}{\delta_{\mathrm{J}}}
$$

substituting from Eqs. (35) through (38) into (34) and then (33), assuming $w_{w}$ $\simeq$ W [12], rearranging gives:

$$
\Delta \mathrm{P}_{s}=\frac{2^{6-2 n}}{\mathrm{R}_{\mathrm{e}} \delta_{\mathrm{J}}{ }^{n}} \int_{0}^{x} \mathrm{w}^{\mathrm{n+1}} \mathrm{dx}
$$

Assuming that the tangential component decay function is similar to that given by Hui Li et al [7], Eq. (6), thus :

$$
\log \left(W / W_{0}\right)=-0.01605 x^{0.8}
$$

substituting for $w$, from Eq. (18), into Eq. (39) gives :

$$
\begin{aligned}
& \Delta \mathrm{P}_{s}=\frac{2^{6-2 n} W_{0}{ }^{n+1}}{\operatorname{Re} \delta_{J}{ }^{n}} I(n, x) \\
& I(n, x)=\int_{0}^{x}\left\{e^{0.037(n+1)} x^{0.8}\right\} d x
\end{aligned}
$$

The effect of the non-Netonian behavior on the swirl pressure drop can be deduced from $\mathrm{Eq}$. (41). Thus substituting $n=1$ into $\mathrm{Eq}$. (42) gives $\Delta \mathrm{P}_{\mathrm{s}} \mid \mathrm{N}$ and the non Newtonian-to-Newtonian swirl pressure drop ratio RP is given by :

where

$$
R P=\left.\Delta P_{s}\right|_{N N} /\left.\Delta P_{s}\right|_{N}=2^{2-n} W_{0}^{n-1} \delta_{J}^{(1+n)} J(n, x)
$$

$$
J(n, x)=I(n, x) / I(1, x)
$$

The integral in Eq. (42) has been evaluated numerically for different indices (n) and distances $(x)$. The corresponding ratio parameter $J(n, x)$ fitted the following relations within a maximum deviation of $\pm 5 \%$.

For pscudo plastic fluids $(\mathrm{n}<1)$ :

$$
J(n, x)=1+(1-n)\left(0,011 \times-3.91 * 10^{-5} x^{2}\right) ; \quad 0 \leq n \leq 1
$$

and for dilatent fluids $(n>1)$ :

$$
\mathrm{J}(\mathrm{n}, \mathrm{x})=1+(1-\mathrm{n})\left(0.008 \mathrm{x}-4.76 * 10^{-5} \mathrm{x}^{2}\right) ; \quad 1 \leq \mathrm{n} \leq 2 \quad \text { (44-c) }
$$

It is noticed that $R P=1$ for $n=1$ (i.e. Newtonian or mdr flow).

With $\Delta \mathrm{P}_{\mathrm{s}} / \mathrm{N}$ being given by $\mathrm{Eq}$. (3), it is now possible to generalize this correlation using Eq. (43). Thus, the procedure of determining the wall static pressure at a section of " $x$ " distance from pipe inlet is as follows:

(i) The coefficient $\lambda$ is determined (using either emprical correlations, or experimental data) and hence the term $\Delta \mathrm{P}_{\mathrm{a}}$ in Eq. (2) can be obtained 
(ii) The swirl intensity $S_{0}$ is determined using either of Eqs. (24) or (29).

(ii) For a section at a distance $x$ ', the corresponding intensity " $S$ " is determined from Eq. (6).

(i ) The corresponding $\Delta \mathrm{P}_{\mathrm{a}} \mid \mathrm{N}$ is obtained from Eq. (2). Then Eqs. (43) through (44-c) are used for determining the ratio RP and hence the term $\Delta \mathrm{P}_{\mathrm{s}} \mid \mathrm{NN}$. Thus the static wall pressure drop at that section is given by:

$$
\Delta \mathrm{P}=\lambda(\mathrm{L}-\mathrm{x})+\left(0.745 \mathrm{~S}+4.16 \mathrm{~S}^{2}\right)^{*} \mathrm{RP}
$$

\section{DISCUSSION}

The validity of the above procedure is tested using a wide range of experimental data for both laminar and turbulent swirl flow of power law fluids [12]. Representative comparative confirmations are shown in Figs. (2) through (11).

Figures (2) through (7) show that the turbulent flow results are well correlated using the suggested procedure. Figures (8) and (9) are for laminar flow, at relatively high

Reynolds numbers, and with friction coefficients determined from Eq. (20). On the other hand, Figs. (10) and (11) are for laminar flow at relatively low Reynolds numbers. The solid lines, show the correlation - Eq. (45) predictions using Eq. (20) for obtaining friction coefficients while the dotted lines show predictions using the linear-downstream-part of the experimental pressure gradient for determining $\lambda$. It is seen that the suggested procedure represents well the pressure distributions within an acceptable accuracy. Deviations between correlation predictions and experimental results may also be attributed to the stated assumptions in addition to the experimental errors.

Predictions of (mdr) conditions are shown in Figs. (12) and (13) both with the Newtonian flow case. Figure (12) shows that drag reduction exists in both swirl and pure axial flow. Another plot is shown in Figure (13). The percentage drag reduction for a pipe length $\mathrm{x}$ is calculated from.

$$
\mathrm{a} D R=\frac{\Delta \mathrm{P}_{\mathrm{N}}-\Delta \mathrm{P}_{\text {ndr }}}{\Delta \mathrm{P}_{\mathrm{N}}} \times 100
$$

It is seen that the $\%$ DR for swirl flow is always lower than that for pure axial one. In addition, it is seen that as pipe length $\mathrm{x}$ increases, the $\% \mathrm{DR}$ increases and approaches its value for pure axial flow. This means that in swirl flows, under drag reductin conditions, the gain in energy saving increases as pipe length increases.

\section{CONCLUSION}

Correlations of wall static pressure distribution for tangential tap generated swirl flow through pipes are given. The correlations require knowledge of the fluid rheological properties as well as the conventional-axial flow-friction factor relations. Expressions for swirl intensity evaluation are given in terms of the 
prevailing geometrical, rheological and flow parameters. These correlations represented reasonably the experimental data for a power law fluid flow in the following ranges Flow behaviorindex $0.62 \leq n \leq 1.0$; flow Reynolds number 200 $\leq R e \leq 21000 ;$ tap to pipe diameter ratio $\mathrm{D}_{\mathrm{J}} / \mathrm{D}=0.339$ and tap distance from wall $\delta_{3} / R=0.339$ and 0.5 .

Application on drag reducing fluid flow under conditions of maximum drag reduction (mdr) shows that, the reduced pressure drop observed for pure axial flow is also observed under swirl flow. Also, the percentage drag reduction increases, as the pipe-length increases, up to its value for pure axial flow where the swirl sensibly decays.

\section{REFERENCES}

1. Frank Kreith and Sonju, O.K., "The decay of turbulent swirl in a pipe"; J. Fluid Mech., vol 22, pt. 2., pp 257-271, 1965.

2. El-Shorbagy, K.A., "Analysis of unguided swirl flow through circular pipes", MSc. Thesis, Faculty of Engg., Alexandria Univ., 1969.

3 Yanjik K.S., and Subbaiah, M.V., "Experiments on swirling turbulent flows"; J. Fluid Mech., vol 60, pt 4, pp 665-687, 1973.

4. Mitsukiyo Murakami, Osami Kito, Yutaka Katayama and Yoshihiko Iida, "An experimental study of swirling flow in pipes", Bull. JSME, V. 19, No 128, pp. 118-126, Feb. 1976.

5. Salem, E.A. El Shorbagy, K.A., "An investigation into the characteristics and decay of swirl flow in pipes"; The Bulletin of the Faculty of Engg., Alex. Univ., Vol. XIX, No, 1, pp 205-224, 1980.

6. Osami Kitoh, "Experimental study of turbulent swirling flow in a straight pipe", J. Fluid Mech., V. 225, pp 445-479, 1991.

7. Hui $\mathrm{Li}$ and Yuji Tomita, "Characteristics of swirling flow in a circular pipe", Trans. ASME, J. Fluids. Engg., V. 116,pp. 370-373, 1994.

8. Parchen, R.R. and Steenbergen,W., "An experimental and numerical study of turbulent swirling pipe flows"; Trans. ASME, J. Fluids Eng., V. 120, pp. 5461, March, 1998.

9. Philip Beran, S., and Fred Culick, E.C., "The role of non-uniqueness in the development of vortex break down in tubes", J. Fluid Mech., V. 242, pp. 491-527, 1992.

10. Wang, S., and Rusak, Z., "The dynamics of a swirling flow in a pipe and transition to axisymmetric vortex break down", J. Fluid Mech., V. 340, pp. 177-223, 1997.

11. Dostal , M., Zitny, R., and Sastak, J., "Helical flow of power law fluids", AIChE J. Vol. 39, No. 1, pp. 189-192, Jan. 1993.

12 Salem Hamdy, "Analysis of non-Newtonian fluids under unguided swirl flow conditions", MSc Thesis, Faculty of Eng., Alexandria Univ., Egypt, 1975.

13. Embaby, M.H., "Detachment of a turbulent plane jet from an inclined plate", Alex. Eng. J., V 35, No. 5, pp. A247-A256, Sept. 1996.

14 Skelland, A.H.P., "Non-Newtonian flow and heat transfer", N.Y., John Wiley, 1967. 
15 Virk, P.S., "Drag reduction fundamentals", AIChE, J., V. 21, pt. 4, pp. 625656, 1975.

16. Bird, R.B., Stewart, W.E. and Lightfoot, E.N., "Transport Phenomena"; John. Wiley \& Sons, inc., N.Y. 1960.

\section{NOMENCLATURE}

$A, A_{0} \quad$ coefficients in Eqs. (10) and (21);

A1 parameter, Eq. (24);

a, aj, $A_{w}$ pipe cross sectional area, tap jet area and wall area $\left(\mathrm{m}^{2}\right)$;

$B, B_{0}$

$\mathrm{B}_{1}$

$\mathrm{D}, \mathrm{D}_{3}$

f

$\mathrm{G}_{1}, \mathrm{G}_{2}$

$\mathrm{G}$

coefficients Eqs. (10) and (21)

parameter, Eq. (24) ;

pipe diameter $(\mathrm{m})$; tap diameter $(\mathrm{m})$;

fanning friction factor $\left(f=\tau_{\mathrm{w}} /\left(0.5 \mathrm{pu}_{\mathrm{in}}^{2}\right)\right)$;

parameters, Eq. (21);

$\mathrm{J}(\mathrm{n}, \mathrm{x}) \quad$ dimensionless parameter, Eq. (44);

$\mathrm{L}, \mathrm{L}$

$\mathrm{n}$

pipe length, dimensionless pipe length (L'/D);

$P^{\prime}, \Delta P^{\prime}, \Delta P$

flow behavior index for a power law fluid;

static pressure $(\mathrm{Pa})$; static pressure difference $=\mathrm{P}_{\mathrm{x}}{ }_{\mathrm{x}}-\mathrm{P}_{\mathrm{c}}{ }_{\mathrm{c}}$;

dimensionless press difference $=\Delta \mathrm{P} /\left(.5 \mathrm{pu}_{\mathrm{m}}^{2}\right)$;

$\Delta \mathrm{P}_{\mathrm{m}} \Delta \mathrm{P}_{\mathrm{s}} \quad$ dimensionless pressure drop due to axial, swirl flow respectively;

$r^{\prime}, R, r \quad$ radial coordinate $(m)$, pipe radius $(m)$, dimensionless $r=r^{\prime} / R$;

$\mathrm{Re}, \mathrm{RP} \quad$ Reynolds number $\mathrm{Eq}(19)$, ratio of non-Newtonian to Newtonian swirl pressure drop $\quad\left(R P=\Delta \mathrm{P}_{\mathrm{s}}\left|\mathrm{NN} / \Delta \mathrm{P}_{\mathrm{s}}\right|_{\mathrm{N}}\right)$;

$S, S_{0} \quad$ swirl intensity Eq. (5), its value at pipe inlet;

$u^{\prime}, u_{m}, u^{*}$ axial velocity component, mean velocity, wall shear velocity $=$ $\sqrt{\tau_{w} / \rho} \quad(\mathrm{m} / \mathrm{s})$;

$u, u^{+}, u_{5} \quad$ dimensionless velocity component $u^{\prime} / u_{m}, u^{\prime} / u^{*}$, characteristic velocity [Eq. (32)];

$\mathrm{w}^{\prime}, \mathrm{W}^{\prime} \quad$ tangential velocity component, the maximum value of this component at a certain section $(\mathrm{m} / \mathrm{s})$;

$\mathrm{w}, \mathrm{W} \quad$ dimensionless velocity $\mathrm{w}=\mathrm{w} / \mathrm{u}_{\mathrm{m}}, \mathrm{W}=\mathrm{W} / \mathrm{u}_{\mathrm{m}}$;

$\mathrm{V}_{\mathrm{J}}, \mathrm{V}_{\mathrm{J}} \quad$ tap jet velocity at pipe inlet $(\mathrm{m} / \mathrm{s})$; dimensionless $=\mathrm{V}_{3}{ }_{3} / \mathrm{u}_{\mathrm{m}}$

$x^{\prime}, x \quad$ axial coordinate $(m)$; dimensionless $x=x^{\prime} / D$;

$\mathrm{y}^{\prime}, \mathrm{y}, \mathrm{y}^{+} \quad$ distance from pipe wall $(\mathrm{m})$, dimensionless distance $\mathrm{y}=\mathrm{y}^{\prime} / \mathrm{R}$, dimensionless distance defined in Eq. (10);

$Z_{1}, Z_{2} \quad$ parameters in Eq. (26) .

\section{Greek Letters:}

$\alpha \quad$ parameter $\mathrm{Eq}(7)$;

$\beta \quad$ parameter $\mathrm{Eq}(7)$;

$\delta^{\prime}, \delta_{\mathrm{J}} \quad$ tap jet center line distance from pipe wall (m), dimensionless

$\delta_{\mathrm{J}}=\delta_{\mathrm{J}} / \mathrm{R}$;

$\Delta_{0 \mathrm{r}} \quad$ deformation tensor component;

$\eta, \eta_{0} \quad$ equivalent viscosity $\left(\mathrm{P}_{\mathrm{a}} \mathrm{s}\right)$, consistency index for a power law 
tap jet inclination angle (deg.);

$\lambda \quad$ friction coefficient $(\lambda=4 \hat{1})$;

$\mu \quad$ Newtonian fluid viscosity (Pa.s)

$\rho \quad$ fluid density $\left(\mathrm{Kg} / \mathrm{m}^{3}\right)$;

$\tau_{\mathrm{w}}, \tau_{\theta \mathrm{r}}, \tau_{\mathrm{w}}$ tangential component of wall shear stress, component of shear stress tensor, wall shear stress $(\mathrm{Pa})$;

$\phi \quad$ dimensionless jet centerline-pipe center line distance Eq. (25)

\section{Subscripts}

e

mdr.

$\mathrm{N}$

$\mathrm{NN}$

o

w

evaluated at pipe end (or exit ) i.e. $x=L$;

Evaluated for conditions of maximum drag reduction;

evaluated for Newtonian fluid;

evaluated for non-Newtonian fluid;

evaluated at pipe inlet i.e at $x=0$;

evaluated at pipe wall;

evaluated at distance $\mathrm{x}$ from pipe inlet. 


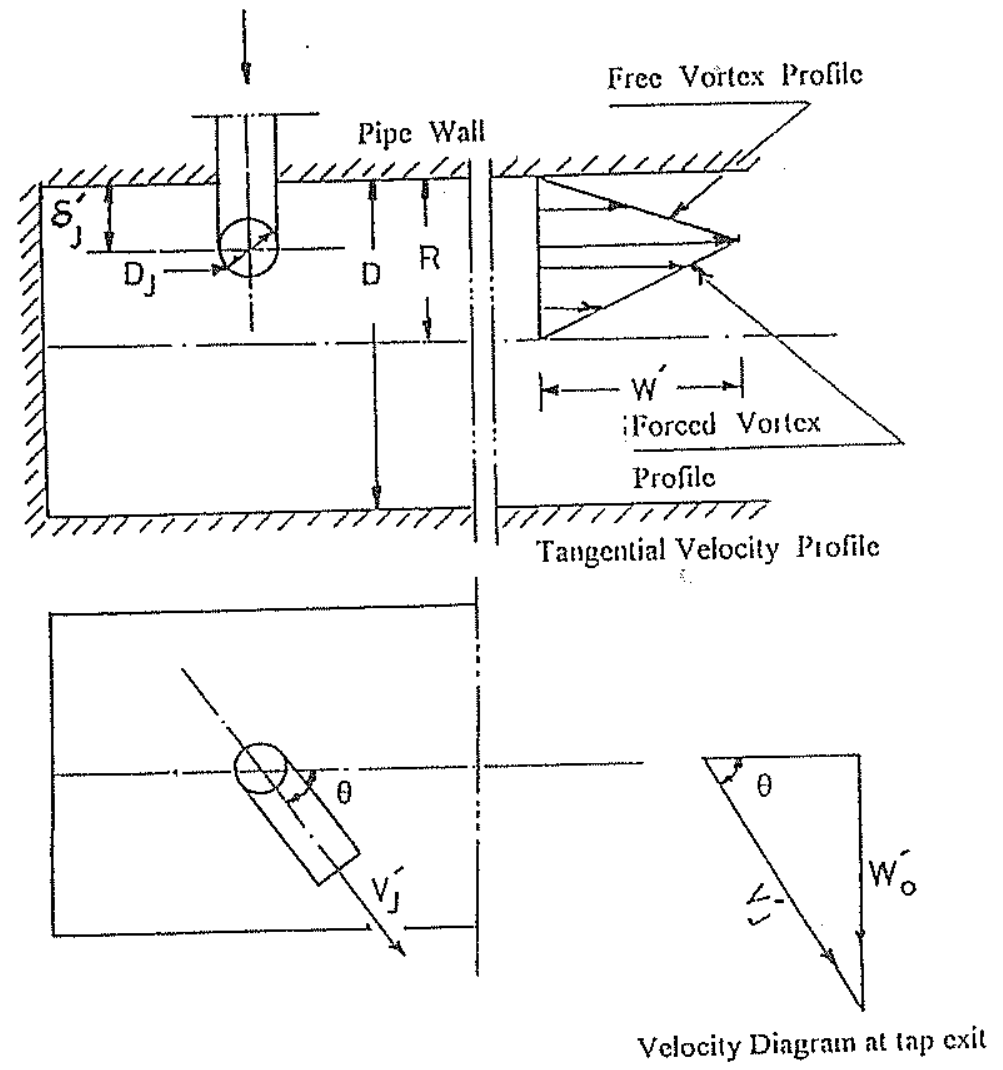

Figure (1) Gcometry of a tangential tap swirl generation system. 


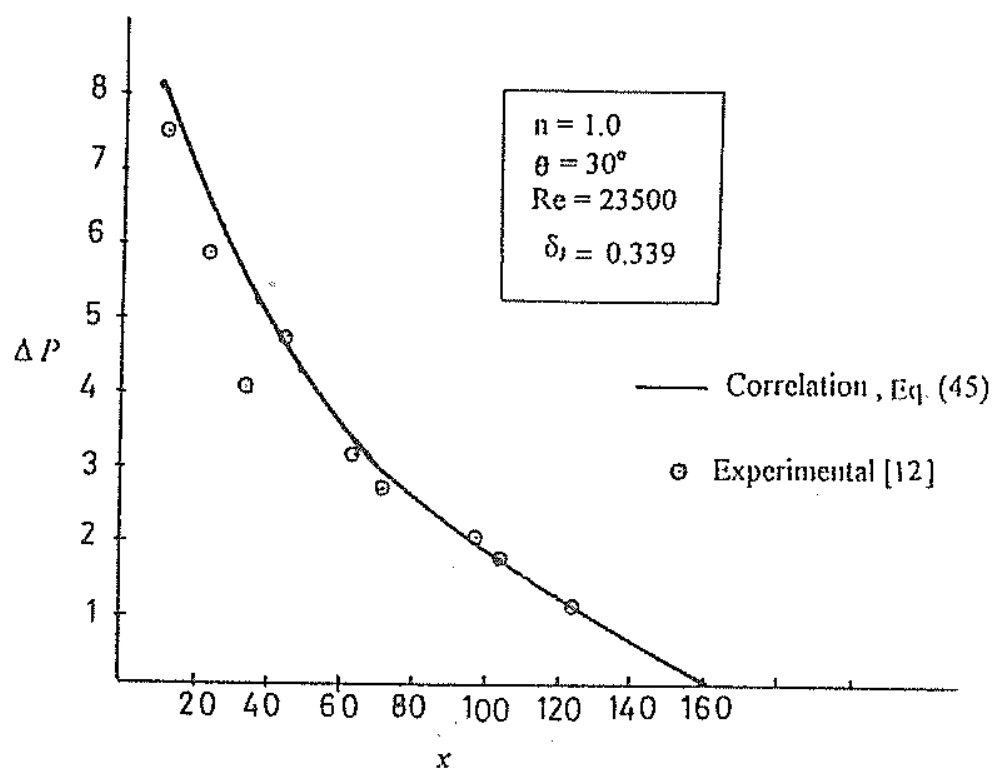

Figure (2) Comparison Between Correlations and Experimental Results

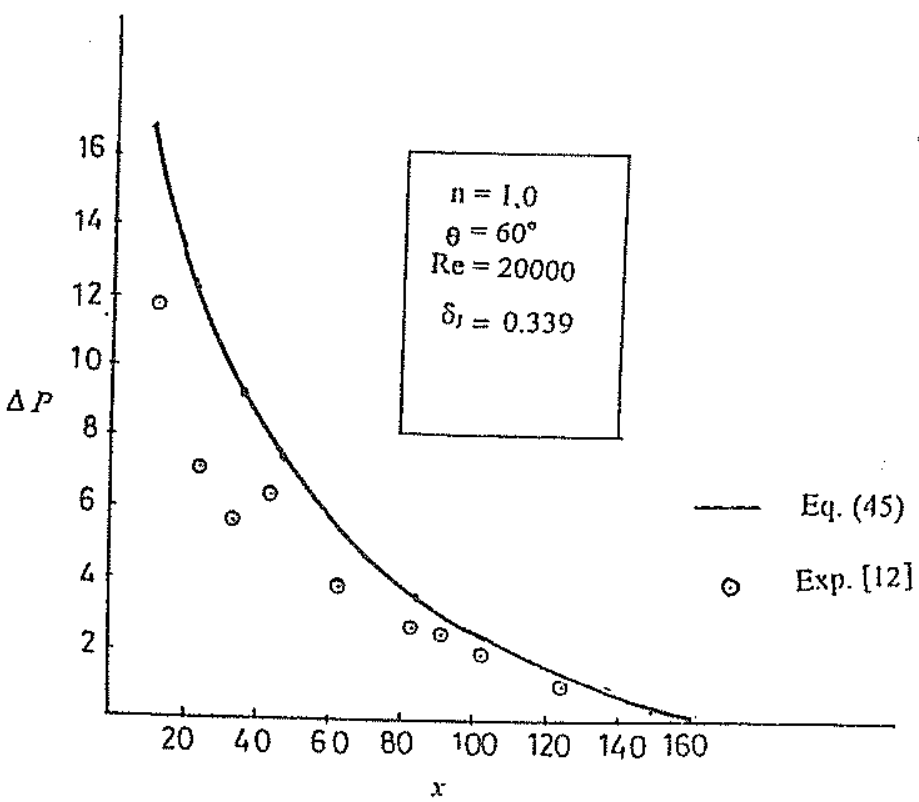

Figure (3) Contd. 


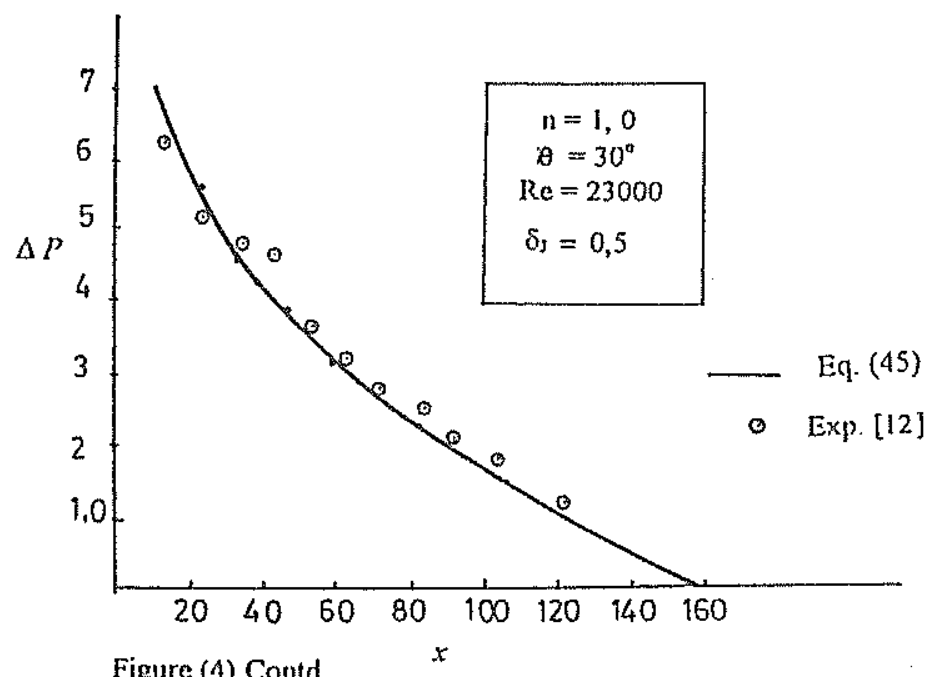

Figure (4) Contd.

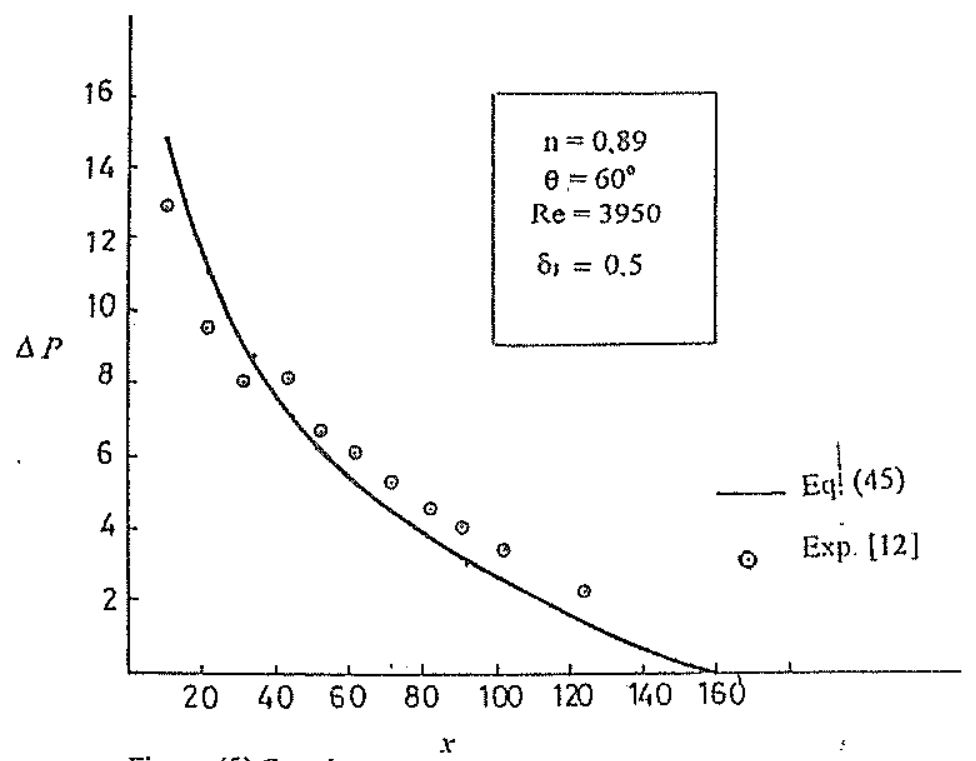

Figure (5) Contd. 


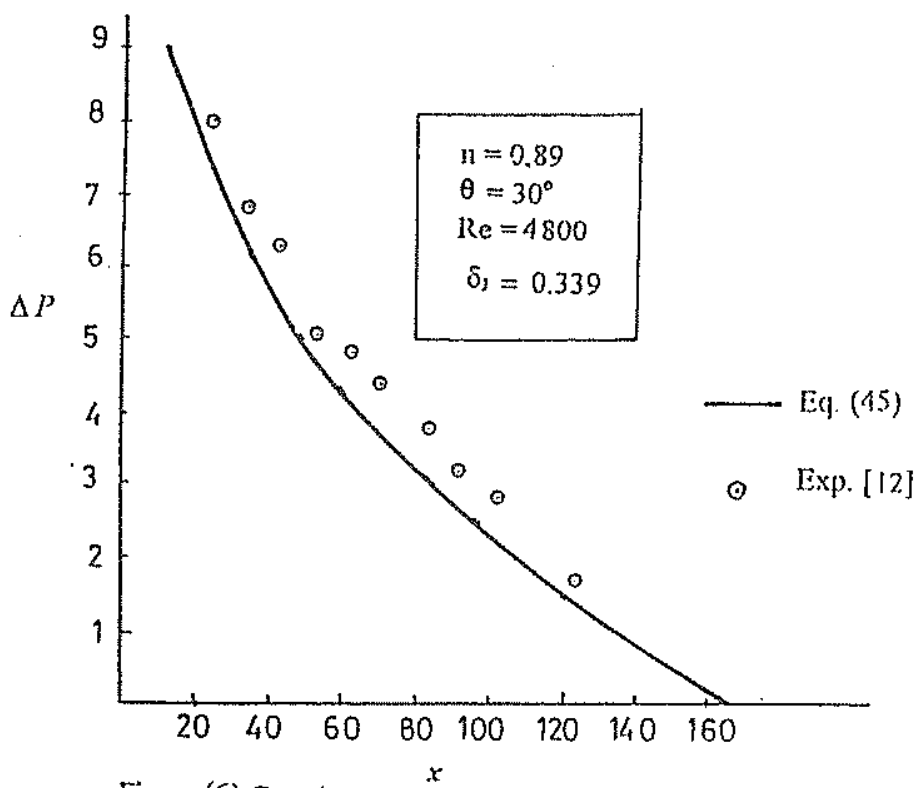

Figure (6) Contd.

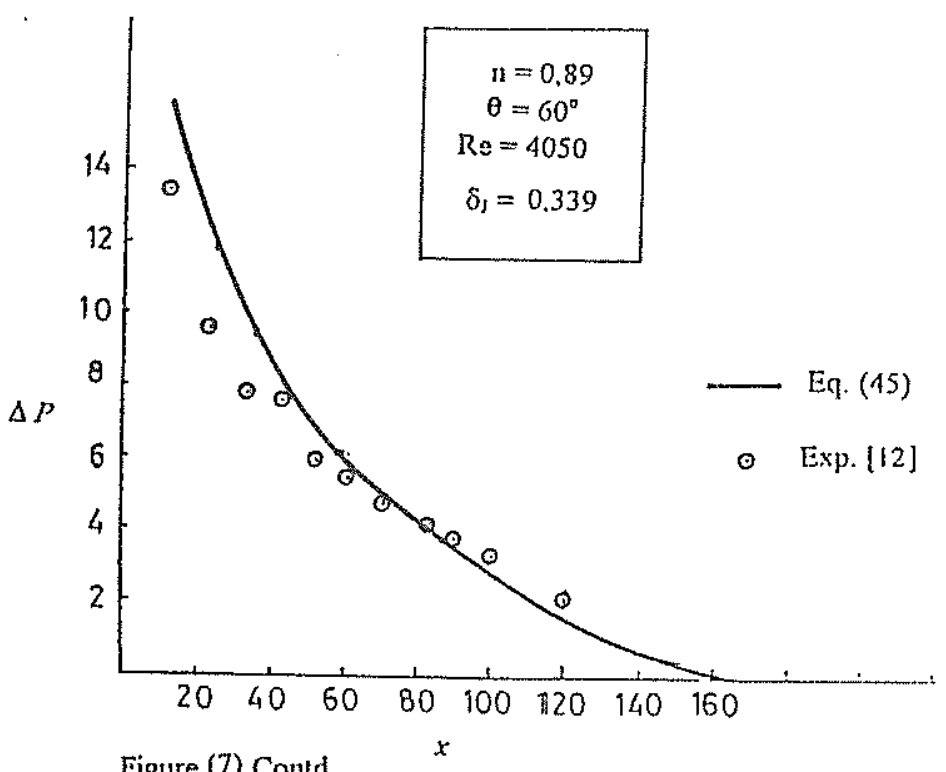

Figure (7) Contd. 


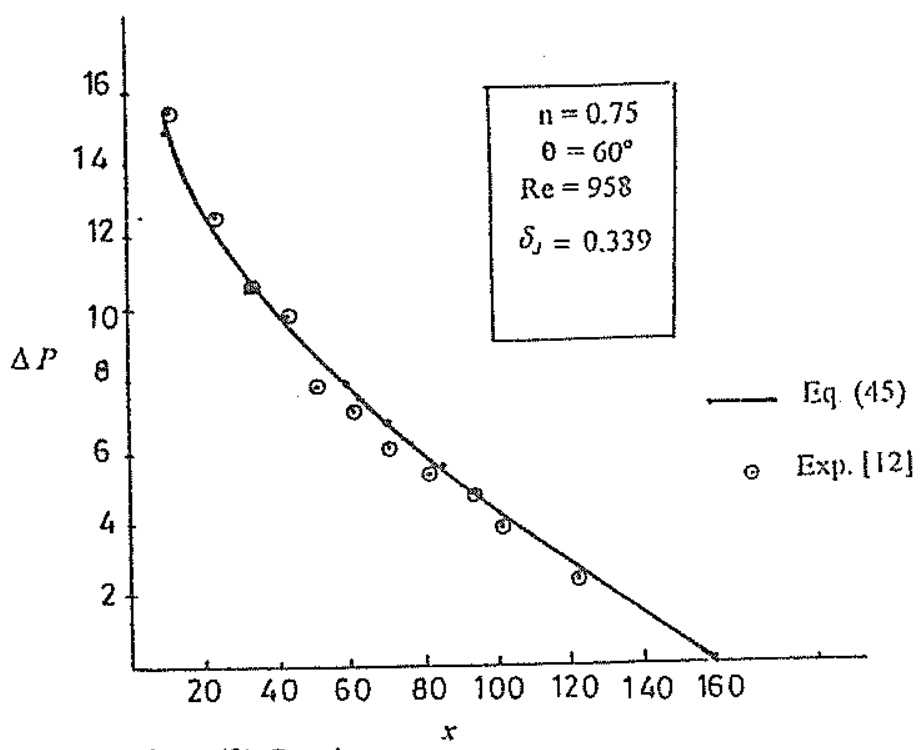

Figure (8) Contd.

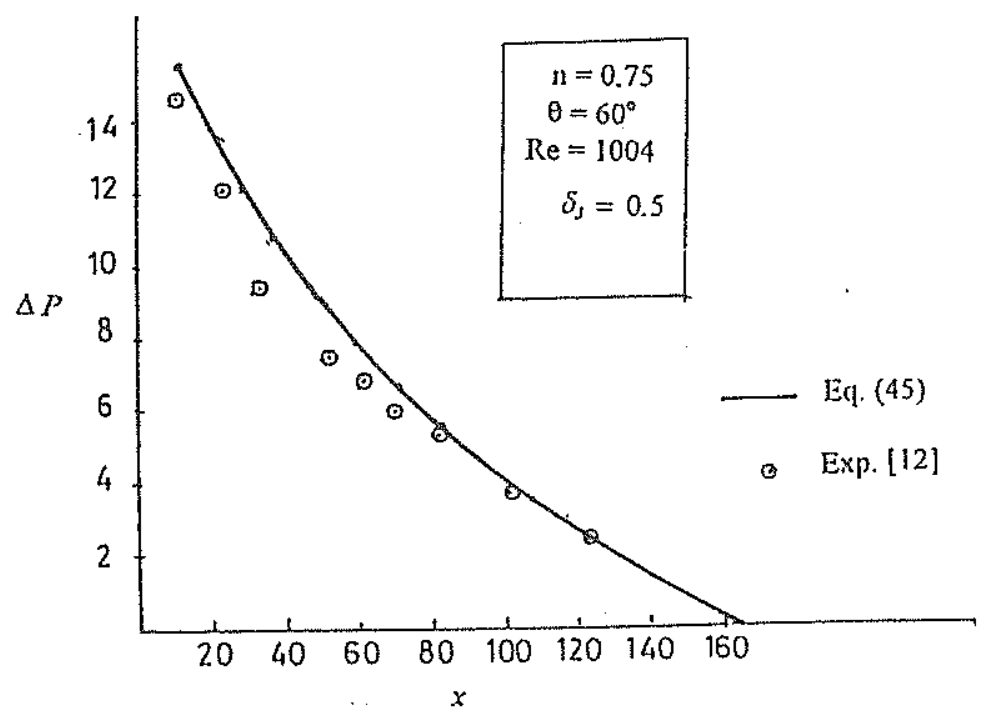

Figure (9) Contd. 


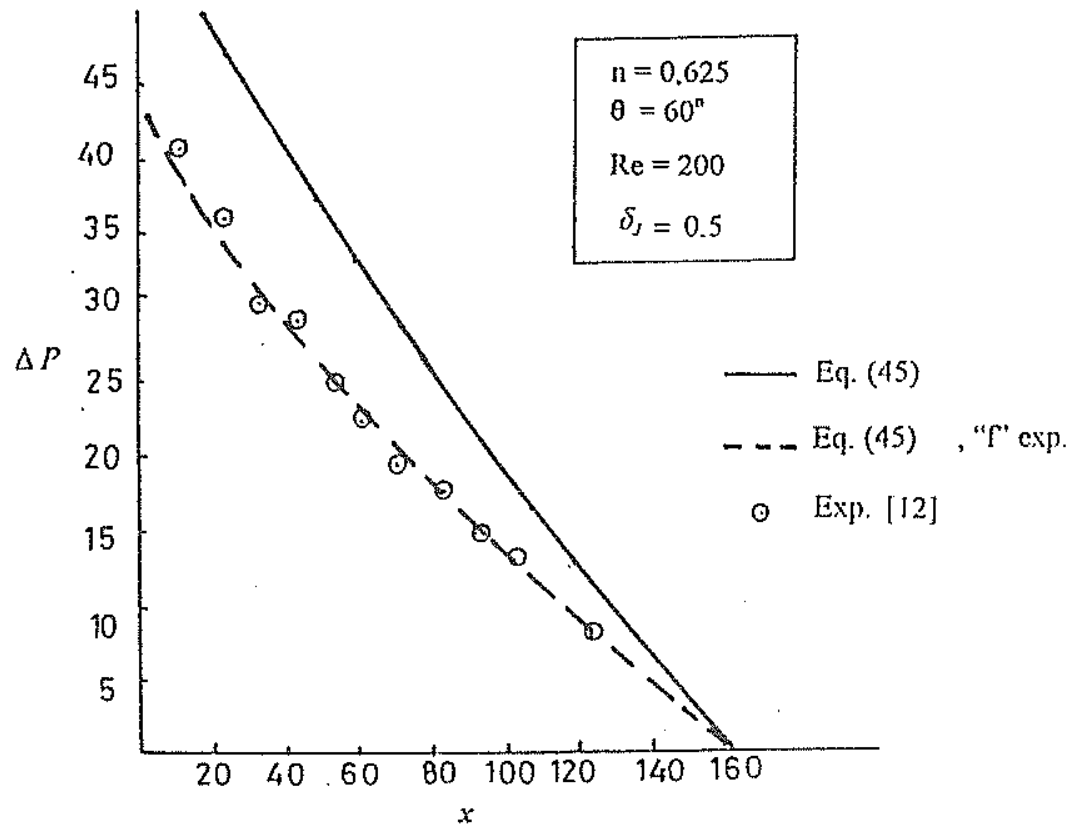

Figure (10) Cond. , friction factor is determined experimentally.

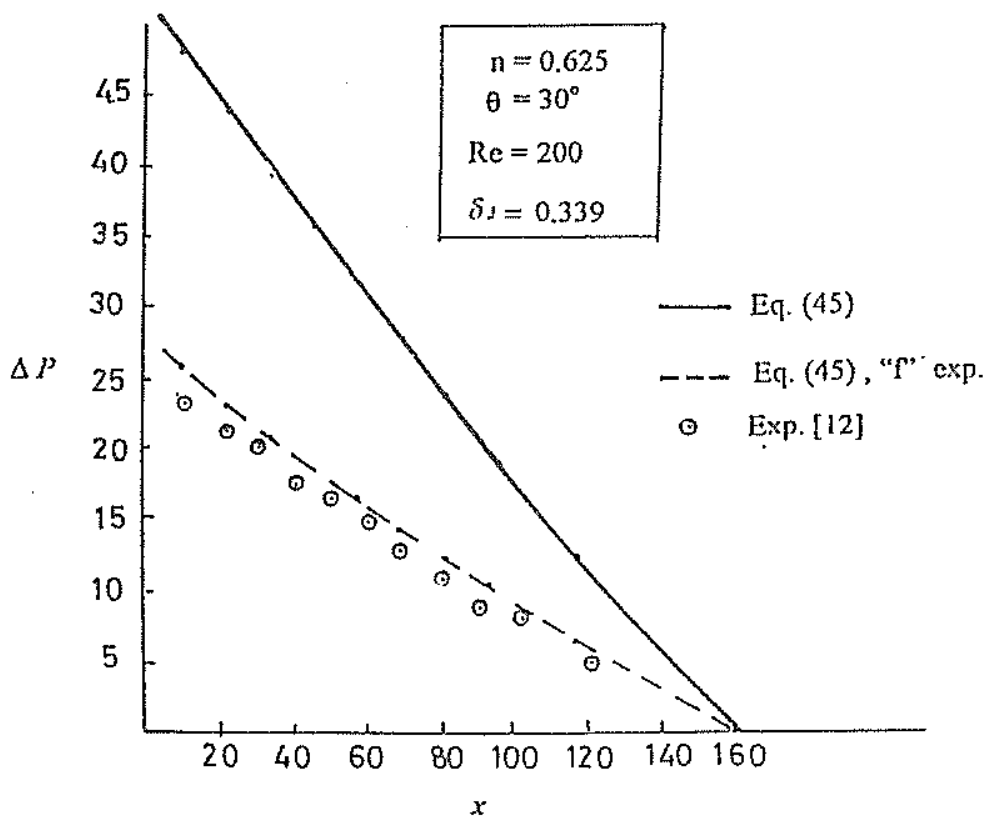

Figure (11) Contd 


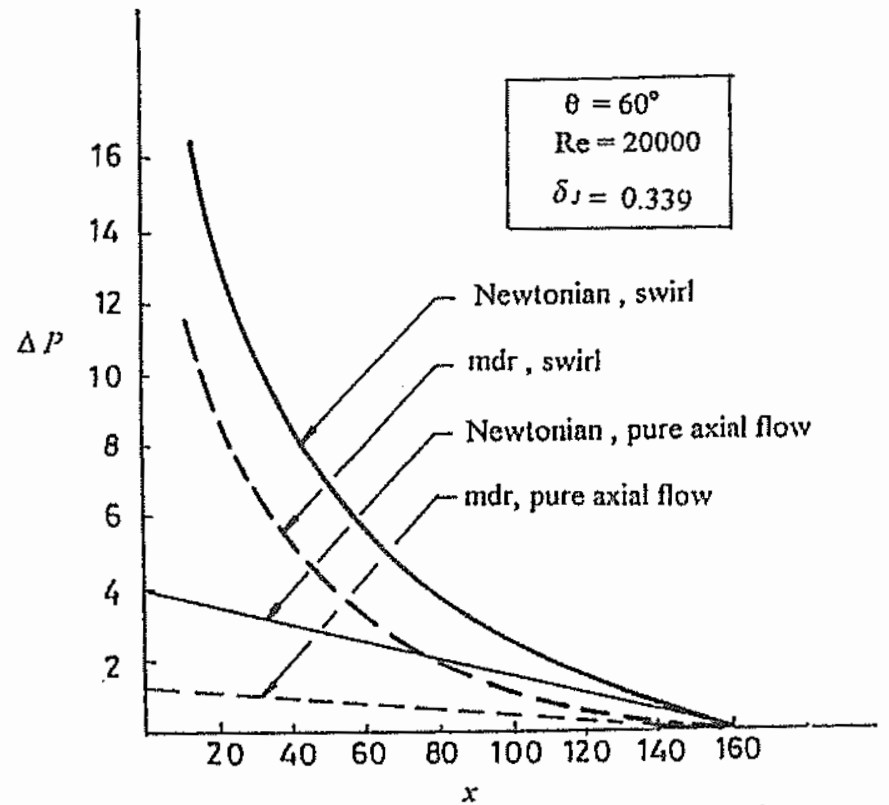

Figure (12) Effect of drag reduction on pressure drop.

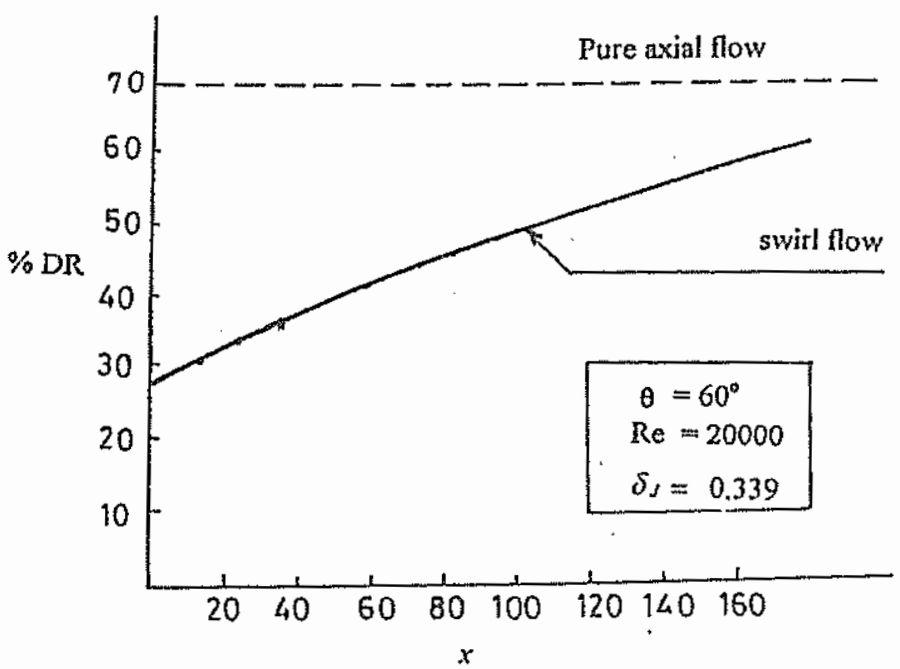

Figure (13) Effect of swirl on drag reduction along a pipe. 


\section{بلخص البهين}

\section{General Correlation of Pressure Distribution for Swirl Flow in Pipes}

M. H. Embaby

Mech. Power Eng. Dept. Faculty of Eng., Menufia Univ., Egypt

(الدالة العامة لتمزيع الضضط للسريان اللدامى فى الأمابيب)

وجد - من الأبعاث إلسابقة - أن هناك دالة لتوزيـع الضغنط على طبوال الأنسابيب

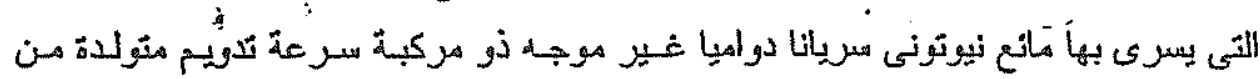

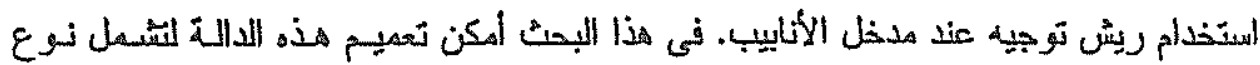

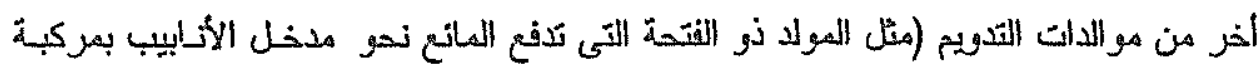

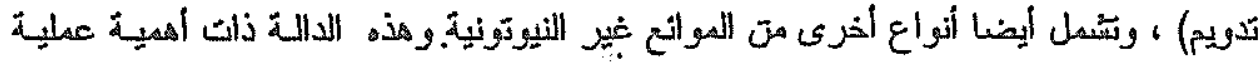

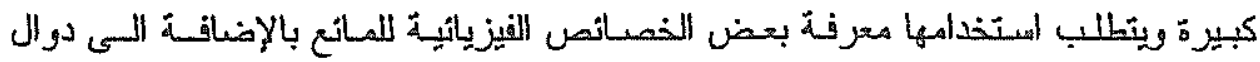

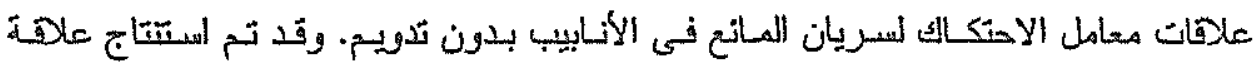

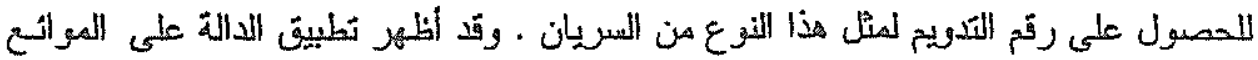

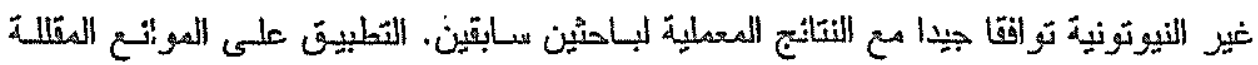

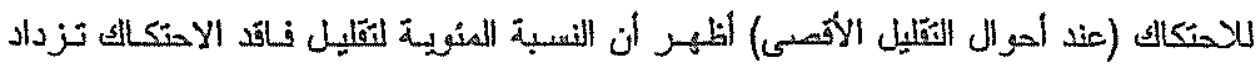

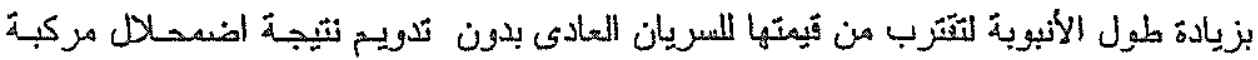

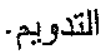

